Elect. Comm. in Probab. 2 (1997) 1-11

ELECTRONIC

COMMUNICATIONS in PROBABILITY

\title{
SURFACE STRETCHING FOR ORNSTEIN UHLENBECK VELOCITY FIELDS
}

\author{
RENE A. CARMONA, STANISLAV GRISHIN, LIN XU \\ Statistics 86 Operations Research Program, CEOR, \\ Princeton University, Princeton N.J. 08544, USA \\ e-mail: rcarmona@princeton.edu \\ sgrishin@chelsea.princeton.edu \\ lin@chelsea.princeton. edu \\ STANISLAV MOLCHANOV \\ Department of Mathematics, \\ University of North Carolina at Charlotte \\ Charlotte, NC 28223 \\ e-mail: smolchan@uncc.edu
}

submitted May 22, 1996; revised September 151996

AMS 1991 Subject classification: 60H10 (60H30)

Keywords and phrases: Diffusion Processes, Lyapunov Exponent, Stochastic Flows.

\begin{abstract}
The present note deals with large time properties of the Lagrangian trajectories of a turbulent flow in $\mathbb{R}^{2}$ and $\mathbb{R}^{3}$. We assume that the flow is driven by an incompressible time-dependent random velocity field with Gaussian statistics. We also assume that the field is homogeneous in space and stationary and Markovian in time. Such velocity fields can be viewed as (possibly infinite dimensional) Ornstein-Uhlenbeck processes. In d spatial dimensions we established the (strict) positivity of the sum of the largest $d-1$ Lyapunov exponents. As a consequences of this result, we prove the exponential stretching of surface areas (when $d=3$ ) and of curve lengths (when $d=2$ ) which confirms conjectures found in the theory of turbulent flows.
\end{abstract}

\section{Introduction}

The mathematical model which we consider is commonly used for the time evolution of a collection of light pollutants carried by a turbulent flow. These pollutants do not affect the flow and can be viewed as passive tracers. To track the motion of a blob of pollutants, we only need to follow the boundary. This boundary is deformed by the flow and its shape can become very involved at times. It is conjectured that, in many cases, the surface area of these boundaries should grow exponentially with time when the flow is turbulent. See for example $[12]$.

We now precise our assumptions on the flow. We assume that it is driven by a mean zero, 
time-stationary and space homogeneous random velocity field $\left\{\overrightarrow{\mathbf{v}}(t, x) ; t \geq 0, x \in \mathbb{R}^{d}\right\}$ and we assume that this field is Gaussian. Hence, its distribution is completely determined by its covariance function:

$$
\mathbb{E}\{\overrightarrow{\mathbf{v}}(s, x) \otimes \vec{v}(t, y)\}=\Gamma(t-s, y-x), \quad s, t \geq 0, x, y \in \mathbb{R}^{d}
$$

or equivalently (provided we assume a mild decay of the correlations in order to garantee integrability of the covariance function) by its spectral density:

$$
E(\omega, k)=\frac{1}{(2 \pi)^{d+1}} \int_{\mathbb{R}} \int_{\mathbb{R}^{d}} e^{i(\omega t+k \cdot x)} \Gamma(t, x) d t d x .
$$

Since $\Gamma(t, x)$ is a $d \times d$ matrix for each $(t, x), E(\omega, k)$ is also a $d \times d$ matrix and the above Fourier transform has to be understood entry by entry. We are concerned with incompressible models, so we assume that the divergence of the velocity field is identically zero. Moreover, we shall assume that it is also isotropic and Markovian in time. These three properties imply that the entries of the spectral density matrix are necessarily of the form:

$$
E_{l, m}(\omega, k)=\frac{\beta(|k|)}{\omega^{2}+\beta(|k|)^{2}} \mathcal{E}(|k|) \frac{1}{|k|^{d-1}}\left(\delta_{l, m}-\frac{k_{l} k_{m}}{|k|^{2}}\right) .
$$

for some nonnegative functions $\beta=\beta(r)$ and $\mathcal{E}(r)$. This form of the spectral density shows that the velocity field can be viewed in the Fourier domain as a superposition of OrnsteinUhlenbeck (OU for short) processes parametrized by the Fourier mode variable $k$. In this interpretation, the quantity $\beta(|k|)$ is the inverse of the correlation length of the OU process associated to the Fourier mode $k$ and the function $\mathcal{E}(k)$ control the spatial correlation of these individual OU processes. The function $\beta=\beta(r)$ is usually chosen of the form $\beta(r)=a r^{z}$ (see for example $[2,3]$ ) while, because of the scaling theory of Kolmogorov's turbulence, the function $\mathcal{E}(r)$ is chosen to be of the form:

$$
\mathcal{E}(r)=r^{1-\epsilon} \quad \text { for } \quad r \in\left[r_{0}, r_{1}\right] \quad \text { and } 0 \text { otherwise. }
$$

Notice that striclty speaking, scaling arguments force the spectral function $\mathcal{E}(|k|)$ to be a power and since a power function cannot be integrable at the origin and at infinity simultaneously, the spectrum (and consequently the velocity field as well) is singular and the cut off limiting the support of the spectral function $\mathcal{E}$ to the interval $\left[r_{0}, r_{1}\right]$ is here to regularize the situation and to make sure that the velocity field is a bona fide field. Following [1], the velocity field could be viewed as a Ornstein-Uhlenbeck process taking values in an infinite dimensional function space. As explained in [8] the crucial operators would then be $A=\beta\left((-\Delta)^{1 / 2}\right)$ and $B=\mathcal{E}\left((-\Delta)^{1 / 2}\right)$ as defined by the functional calculus of self-adjoint operators. We are not able to work at this level of generality and we shall restrict our study to the velocity fields which we used for computer simulations and which are given by spectra with finitely many Fourier modes (i.e. with a point measure with finite support instead of the spectral measure $\mathcal{E}(|k|) d k$.

The incompressibility of the d-dimensional vector field $\overrightarrow{\mathbf{v}}(t, x)$ can be equivalently stated by assuming the existence (for each $(t, x))$ of a $d \times d$ antisymmetric (random) matrice $\left[\mathcal{H}_{i j}(t, x)\right]_{i, j}$ whose entries are differentiable and such that the components of the velocity field satisfy:

$$
v_{i}(t, x)=\sum_{j=1}^{d} \frac{\partial \mathcal{H}_{i j}(t, x)}{\partial x^{j}}, \quad i=1, \cdots, d .
$$


In dimension $d=3$ this means that:

$$
\overrightarrow{\mathbf{v}}=\operatorname{curl} \vec{\phi}
$$

where $\vec{\phi}(t, x)=\left[\phi^{1}\left(t, x^{1}, x^{2}, x^{3}\right), \phi^{2}\left(t, x^{1}, x^{2}, x^{3}\right), \phi^{3}\left(t, x^{1}, x^{2}, x^{3}\right)\right]$ is a 3 dimentional vector field and in $d=2$ dimensions it means the existence of a stream function $\phi$ for which:

$$
\overrightarrow{\mathbf{v}}(t, x)=\nabla^{\perp} \phi(t, x)=\left[\begin{array}{c}
\frac{\partial \phi(t, x)}{\partial x^{2}} \\
-\frac{\partial \phi(t, x)}{\partial x^{1}}
\end{array}\right] .
$$

The results we are interested are well known in the case of Brownian flows corresponding to a covariance $\Gamma(t, x)$ of the form $\delta_{0}(t) Q(x)$. They were proved by Le Jan in [16] and generalized in [7] to the case of compact manifolds. As explained earlier, we are not able to handle the general case described above and we assume that the problem is finite dimensional in nature. As in [16], [17] and [7] this makes it possible to use probabilistic diffusion theory. The second advantage is that the model has its own interest due to the fact that the model is the one used in the computer simulations and modeling.

Since the details of the model have been spelled out several times already in the two-dimensional case (see for example [8] or [9] and [11]) we shall concentrate on the case $d=3$. We assume that:

$$
\vec{\phi}(t, x)=\sum_{k \in \mathcal{K}}\left[\vec{a}_{k}(t) \cos (k \cdot x)+\vec{b}_{k}(t) \sin (k \cdot x)\right]
$$

where $\mathcal{K} \subset \mathbb{R}^{3}$ is finite and the $\left\{\vec{a}_{k}(t) ; t \geq 0\right\}$ and the $\left\{\vec{b}_{k}(t) ; t \geq 0\right\}$ are independent 3 -dimensional OU processes-solutions of the stochastic differential system:

$$
\begin{aligned}
d \vec{a}_{k}(t) & =-C_{k} \vec{a}_{k}(t) d t+\Sigma_{k} d \vec{w}_{k} \\
d \vec{b}_{k}(t) & =-C_{k} \vec{b}_{k}(t) d t+\Sigma_{k} d \vec{w}^{\prime}{ }_{k}
\end{aligned}
$$

where $C_{k}$ and $\Sigma_{k}$ are $3 \times 3$ matrices called the drift matrix and the diffusion matrix respectively. and where the processes $\overrightarrow{w_{k}}$ and $\overrightarrow{w_{k}^{\prime}}$ are independent 3-dimensional standard Wiener processes. We shall assume that $C_{k}$ is positive definite. The velocity field is then defined by $\overrightarrow{\mathbf{v}}=\operatorname{curl}(\vec{\phi})$. The set of Fourier modes $\mathcal{K}$ and the coefficient matrices $C_{k}$ and $\Sigma_{k}$ should be chosen as specific functions of the functions $\beta$ and $\mathcal{E}$ introduced above in our discussion of the Kolmogorov's spectrum. The purpose of that choice is to mimick the spectral properties described earlier. See for example [9] for details on the 2-dimensional case. Each pollutant particle position, say $x_{t}$, satisfies the equation of motion:

$$
\left\{\begin{array}{l}
d x_{t}=\overrightarrow{\mathbf{v}}\left(t, x_{t}\right) d t \\
x_{0}=x
\end{array}\right.
$$

if it starts from $x$ at time $t=0$. Setting $\varphi_{0, t}(x)=x_{t}$ defines a semigroup of diffemorphisms of $\mathbb{R}^{3}$. Let us assume for example that the initial position of the blob of pollutants is the volume enclosed in a smooth surface $S_{0}=\{S(\alpha, \beta) ; 0 \leq \alpha \leq 1,0 \leq \beta \leq 1\}$. Then at each later time $t$ the blob is enclosed in $S_{t}=\left\{\varphi_{0, t}(S(\alpha, \beta)) ; 0 \leq \alpha \leq 1,0 \leq \beta \leq 1\right\}$. The main thrust of the paper is to determine the large time asymptotics of the surface area of $S_{t}$. We shall denote the latter by $\left|S_{t}\right|$ or $\left|\varphi_{0, t}(S)\right|$. The main result is summarized as the following theorem

Theorem 1.1 If the velocity field $\overrightarrow{\mathbf{v}}(t, z)$ is as above and if the linear span in $\mathbb{R}^{3}$ of the set $\mathcal{K}$ of Fourier modes is equal to the whole $\mathbb{R}^{3}$, then the surface area $\left|S_{t}\right|=\left|\phi_{0, t}\left(S_{0}\right)\right|$ grows exponentially as $t \rightarrow \infty$. 


\section{Remark:}

- The condition on $\mathcal{K}$ is necessary for the above exponentially growth to hold. Indeed in the two dimensional case of a shear flow, $\mathcal{K}$ is a subset of a line and it is possible to check directly that the length does not grow exponentially (see [8]).

- It is straightforward to extend this result to higher dimensional spaces.

The proof has three main components. 1) Reduction of the problem to questions concerning Lyapunov exponents of a random linear system. These exponents have been extensively investigated for systems driven by ergodic Markov processes. There are many nice references on the subject(e.g. see $[18,10,13])$ but the work of Bougerol $([5,6])$ seems to be best suited to our present needs. 2) Proving the properties of the Lagrangian observations which are needed to apply results of [5] and finally 3) Checking the non-degeneracy conditions always present in the analysis of Lyapunov exponents.

This strategy becomes clear if we write:

$$
\left|S_{t}\right|=\int_{[0,1] \times[0,1]}\left|\left[\frac{\partial \varphi_{0, t}(S(\alpha, \beta))}{\partial \alpha} \times \frac{\partial \varphi_{0, t}(S(\alpha, \beta))}{\partial \beta}\right]\right| d \alpha d \beta,
$$

(starting here for the rest of this paper, " $\times$ " stands for the usual vector product) and, differentiating both sides of equation (5):

$$
\begin{cases}d\left(\frac{\partial \varphi_{0, t}(S(\alpha, \beta))}{\partial \alpha}\right)= & \frac{\partial \overrightarrow{\mathbf{v}}}{\partial z}\left(t, \vec{x}_{t}\right) \frac{\partial \varphi_{0, t}(S(\alpha, \beta))}{\partial \alpha} d t \\ \frac{\partial \varphi_{0,0}(S(\alpha, \beta))}{\partial \alpha}= & \frac{\partial S(\alpha, \beta)}{\partial \alpha},\end{cases}
$$

with a similar equation for the partial derivative of $\varphi_{0, t}(S(\alpha, \beta))$ with respect to $\beta$. This shows that the partial derivatives appearing in formula (6) are solutions of the same (random) linear systems with matrix of coefficients equal to $\nabla_{x} \overrightarrow{\mathbf{v}}\left(t, \varphi_{0, t}(x)\right)$. This matrix is the observation of the gradient $\nabla_{x} \overrightarrow{\mathbf{v}}(t, x)$ of the velocity field along the Lagrangian trajectory $t \hookrightarrow x_{t}=\varphi_{0, t}(x)$. This matrix of coefficients is a stationary process in time (see for example [19]) and the subadditive ergodic theorem garantees the existence of Lyapunov exponents.

The rest of the paper is organized as follows. In the next section, we state the results on random linear systems which we need. In the following section we establish the Lagrangian properties of the flow, especially the Markov property and the ergodicity of the relevant processes. In the last section, we prove the main theorem.

The problem addressed here is of interest in different contexts. Indeed, the same issues are considered in [4] for the analysis of the magnetic field of turbulent fluids and especially the dynamo effect, in [12] in the context of vorticity stretching in turbulent fluids and in [8] in the context of mathematical oceanography. But so far, rigorous results have only been established for Brownian flows.

Finally we want to emphasize that our motivation and the choice of the finitely many Fourier modes model were strongly influenced by the results of the computer simulations reported in [8] and [9] for the case $d=2$.

\section{Lyapunov Exponents of Random Linear Systems}

Let $\{Y(t) ; t \geq 0\}$ be a $m$ dimensional ergodic diffusion process and let $A$ be a function from $\mathbb{R}^{m}$ into the space of $d \times d$ real matrices with trace 0 and let us consider the following (random) 
linear system:

$$
\frac{d M_{t}}{d t}=A(Y(t)) M_{t}
$$

in the $d \times d$ unknown matrix $M_{t}$. We assume that the diffusion $Y(t)$ is given by:

$$
d Y(t)=V_{0}(Y(t)) d t+\sum_{i=1}^{m} V_{i}\left(Y_{t}\right) d B_{t}^{i}
$$

where the $V_{j}$ are smooth vector fields on $\mathbb{R}^{m}$ and the $B^{j}$ 's are independent real valued standard Wiener processes. For each element $u$ of $\mathbb{R}^{d}$ we use the notation:

$$
\Theta_{t}^{u}=\frac{M_{t} u}{\left\|M_{t} u\right\|}
$$

for the action of the matrix $M_{t}$ on the unit sphere. Here $\|\cdot\|$ is the usual Euclidean norm in $\mathbb{R}^{d}$. An elementary calculation shows that:

$$
\frac{d \Theta_{t}^{u}}{d t}=A(Y(t)) \Theta_{t}^{u}-\left[\left(\Theta_{t}^{u}\right)^{t} A(Y(t)) \Theta_{t}^{u}\right] \Theta_{t}^{u} .
$$

Consequencely, $\left(Y(t), \Theta_{t}^{u}\right)$ is a (possibly degenerated) diffusion. We quote a well known result concerning the Lyapunov exponents of the above random system. See [5] for a proof.

Lemma 2.1 Let us assume that $M_{t}, Y(t)$ and $\Theta_{t}^{u}$ are as above and that $\left(Y(t), \Theta_{t}^{u}\right)$ is hypoelliptic in the sense that the Lie algebra generated by $V_{0}, \cdots, V_{d}, \frac{\partial}{\partial t},\left[A(y) \theta-\left(\theta^{\prime} A(y) \theta\right) \theta\right] \cdot \frac{\partial}{\partial \theta}$ has dimension $d+m$ everywhere. Then there exist constants $\mu_{1} \geq \cdots \geq \mu_{d}$ and linearly independent (unit) vectors $v_{1}, \cdots, v_{d}$ such that:

$$
\lim _{t \rightarrow \infty} \frac{1}{t} \ln \left|M_{t} v_{1} \wedge \cdots \wedge M_{t} v_{i}\right|=\mu_{1}+\cdots+\mu_{i}
$$

for each $i \leq d$. Here and in the following $\wedge$ denotes the usual exterior product.

The $\mu_{1}, \cdots, \mu_{d}$ are usually refered to as the Lyapunov exponents of the system. The following result from [6] shows that they are different under suitable conditions.

Lemma 2.2 With the same notations, $\mu_{1}>\mu_{d}$ if there does not exist a positive quadradic form $Q(\cdot, \cdot)$ satisfying almost surely:

$$
Q(u, u)=\left(\operatorname{det} M_{t}\right)^{-\frac{2}{d}} Q\left(M_{t} u, M_{t} u\right)
$$

for all $u \in \mathbb{R}^{d}$.

\section{Lagrangian Observations}

We recast (7) in the framework of the previous section. From now on we assume that the starting point $x$ is fixed. Since $\nabla \overrightarrow{\mathbf{v}}\left(t, x_{t}\right)$ is not a Markov process we view it as a function of the Markov process $\left\{\overrightarrow{\mathbf{v}}\left(t, X_{t}+z\right) ; z \in \mathbb{R}^{3}\right\}$. But unfortunately, the latter is infinite dimensional in general. This illustrates our interest in the finitely Fourier modes assumption which reduces the complexity to finite dimensional diffusion processes. We follow the discussion of [11] whose results we recall in the present context. 
For each $k \in \mathcal{K}, \overrightarrow{a_{k}}$ and $\overrightarrow{b_{k}}$ are OU processes in $\mathbb{R}^{3}$. Since the drift matrices $C_{k}$ are positive definite, these OU processes have a unique invariant measure $\nu_{k}(d x)$ given by:

$$
\nu_{k}(d x)=\frac{1}{Z_{k}} e^{-x^{t} D_{k}^{-1} x / 2} d x
$$

where the (covariance) matrix $D_{k}$ is defined by:

$$
D_{k}=\int_{0}^{\infty} e^{-s C_{k}} \Sigma_{k}^{t} \Sigma_{k} e^{-s C_{k}} d s .
$$

As usual we use the denominator $Z_{k}$ as a normalizing constant. If the matrices $C_{k}$ and $\Sigma_{k}$ commute, then

$$
D_{k}=\frac{1}{2} \Sigma_{k}^{t} \Sigma_{k} C_{k}^{-1}
$$

Let us set $m=3 n$ where $n=|\mathcal{K}|$ is the cardinality of $\mathcal{K}$ and let use the notation $X(t)$ for the $\mathbb{R}^{m}$-valued OU process $\left\{\vec{a}_{k}(t), \vec{b}_{k}(t), k \in \mathcal{K}\right\}$. Its invariant measure $\nu$ is given by the product of the $\nu_{k}$ 's. A straightforward computation shows that:

$$
\tilde{\vec{\phi}}(t, z)=\vec{\phi}\left(t, x_{t}+z\right)=\sum_{k \in \mathcal{K}}\left[\tilde{\vec{a}}_{k}(t) \cos (k \cdot z)+\tilde{\vec{b}}_{k}(t) \sin (k \cdot z)\right]
$$

where $\tilde{\vec{a}}_{k}(t)$ and $\tilde{\vec{b}}_{k}(t)$ are defined by:

$$
\begin{aligned}
& \tilde{\vec{a}}_{k}(t)=\vec{a}_{k}(t) \cos \left(k \cdot x_{t}\right)+\vec{b}_{k}(t) \sin \left(k \cdot x_{t}\right) \\
& \tilde{\vec{b}}_{k}(t)=-\vec{a}_{k}(t) \sin \left(k \cdot x_{t}\right)+\vec{b}_{k}(t) \cos \left(k \cdot x_{t}\right) .
\end{aligned}
$$

Since $\overrightarrow{\mathbf{v}}\left(t, x_{t}+z\right)=\operatorname{curl}_{z}\left(\vec{\phi}\left(t, x_{t}+z\right)\right)$ we have:

$$
\begin{aligned}
\tilde{\overrightarrow{\mathbf{v}}}(t, z) & =\overrightarrow{\mathbf{v}}\left(t, x_{t}+z\right) \\
& =\sum_{k \in \mathcal{K}}\left[-\left(\tilde{\vec{a}_{k}}(t) \times k\right) \sin (k \cdot z)+\left(\tilde{\vec{b}_{k}}(t) \times k\right) \cos (k \cdot z)\right] .
\end{aligned}
$$

The definition of the process $Y(t)=\left\{\tilde{\vec{a}}_{k}(t), \tilde{\vec{b}}_{k}(t), k \in \mathcal{K}\right\}$ looks involved at first but we now show that it is a diffusion process.

Lemma 3.1 $Y$ is a diffusion process on $\mathbb{R}^{m}$ with infinitesimal generator

$$
\begin{gathered}
\tilde{\mathcal{L}}=\frac{1}{2} \sum_{k \in \mathcal{K}} \operatorname{tr}\left(\Sigma_{k}^{t} H_{\tilde{a}_{k}} \Sigma_{k}+\Sigma_{k}^{t} H_{\tilde{b}_{k}} \Sigma_{k}\right)-\sum_{k \in \mathcal{K}}\left(C_{k} \tilde{a}_{k} \cdot \nabla_{\tilde{a}_{k}}+C_{k} \tilde{b}_{k} \cdot \nabla_{\tilde{b}_{k}}\right) \\
+\sum_{k, k^{\prime} \in \mathcal{K}} \operatorname{det}\left(\vec{k}, \overrightarrow{k^{\prime}}, \overrightarrow{b_{k^{\prime}}}\right)\left(\tilde{b}_{k} \cdot \nabla_{\tilde{a}_{k}}-\tilde{a}_{k} \cdot \nabla_{\tilde{b}_{k}}\right),
\end{gathered}
$$

where we use the notations:

$$
\begin{aligned}
\nabla_{\tilde{a}_{k}}=\left[\frac{\partial}{\partial \tilde{a}_{k}^{1}}, \frac{\partial}{\partial \tilde{a}_{k}^{2}}, \frac{\partial}{\partial \tilde{a}_{k}^{3}}\right]^{t} & \nabla_{\tilde{b}_{k}}=\left[\frac{\partial}{\partial \tilde{b}_{k}^{1}}, \frac{\partial}{\partial \tilde{b}_{k}^{2}}, \frac{\partial}{\partial \tilde{b}_{k}^{3}}\right]^{t} \\
H_{\tilde{a}_{k}}=\left[\frac{\partial^{2}}{\partial \tilde{a}_{k}^{l} \partial \tilde{a}_{k}^{m}}\right]_{l, m} & H_{\tilde{b}_{k}}=\left[\frac{\partial^{2}}{\partial \tilde{b}_{k}^{l} \partial \tilde{b}_{k}^{m}}\right]_{l, m}
\end{aligned}
$$


for the partial differential operators and:

$$
\operatorname{det}\left(\vec{k}, \overrightarrow{k^{\prime}}, \vec{b}\right)=k^{1}\left(k^{\prime 2} \tilde{b}^{3}-k^{\prime 3} \tilde{b}^{2}\right)-k^{2}\left(k^{\prime 1} \tilde{b}^{3}-k^{\prime 3} \tilde{b}^{1}\right)+k^{3}\left(k^{\prime 1} \tilde{b}^{2}-k^{\prime 2} \tilde{b}^{1}\right)
$$

for the determinant.

Remark: ¿From the form of the infinitesimal generator one sees that the diffusion $Y$ is strongly elliptic.

Proof:

Using (15), (4) and Ito's formula, we get:

$$
\begin{aligned}
& d \overrightarrow{\tilde{\tilde{a}}}_{k}(t)=-C_{k} \overrightarrow{\tilde{\tilde{a}}}_{k}(t) d t+\Sigma_{k} d \overrightarrow{\tilde{\tilde{w}}}_{k}+\overrightarrow{\tilde{b}}_{k}(t)\left(k \cdot d x_{t}\right) \\
& d \overrightarrow{\tilde{b}}_{k}(t)=-C_{k} \overrightarrow{\tilde{b}}_{k}(t) d t+\Sigma_{k} d \overrightarrow{\tilde{w}}^{\prime}{ }_{k}-\overrightarrow{\tilde{a}}_{k}(t)\left(k \cdot d x_{t}\right)
\end{aligned}
$$

where $\left\{\overrightarrow{\tilde{w}}_{k}, \overrightarrow{\tilde{w}}^{\prime}{ }_{k}, k \in \mathcal{K}\right\}$ are independent 3-dimensional standard Wiener processes. On the other hand, from (16) we see that:

$$
\tilde{v}(t)=\overrightarrow{\mathbf{v}}\left(t, \vec{x}_{t}\right)=\sum_{k \in \mathcal{K}}\left[k^{2} \tilde{b}_{k}^{3}(t)-k^{3} \tilde{b}^{2}(t), k^{3} \tilde{b}^{1}(t)-k^{1} \tilde{b}^{3}(t), k^{1} \tilde{b}^{2}(t)-k^{2} \tilde{b}_{k}^{1}(t)\right]^{t}
$$

and as an immediate consequence we get:

$$
\begin{aligned}
& d \overrightarrow{\tilde{a}}_{k}(t)=-C_{k} \overrightarrow{\tilde{a}}_{k}(t) d t+\overrightarrow{\tilde{b}}_{k}(t) \sum_{k^{\prime} \in \mathcal{K}} \operatorname{det}\left(k, k^{\prime}, b_{k}\right) d t+\Sigma_{k} d \overrightarrow{\tilde{w}}^{\prime}{ }_{k} \\
& d \overrightarrow{\tilde{b}}_{k}(t)=-C_{k} \overrightarrow{\tilde{b}}_{k}(t) d t+-\overrightarrow{\tilde{a}}_{k}(t) \sum_{k^{\prime} \in \mathcal{K}} \operatorname{det}\left(k, k^{\prime}, b_{k}\right) d t+\Sigma_{k} d \overrightarrow{\tilde{w}}^{\prime}{ }_{k}
\end{aligned}
$$

This completes our proof.

Next we investigate the ergodic properties of $Y$. As a byproduct of the explicit form of the adjoint of its infinitesimal generator, we get its invariant measure and its dual process.

Lemma 3.2 The adjoint $\tilde{\mathcal{L}}^{*}$ of the operator $\tilde{\mathcal{L}}$ with respect to the probability measure $\nu$ is

$$
\begin{gathered}
\tilde{\mathcal{L}}^{*}=\frac{1}{2} \sum_{k \in \mathcal{K}} \operatorname{tr}\left(\Sigma_{k}^{t} H_{\tilde{a}_{k}} \Sigma_{k}+\Sigma_{k}^{t} H_{\tilde{b}_{k}} \Sigma_{k}\right)-\sum_{k \in \mathcal{K}}\left(C_{k} \tilde{a}_{k} \cdot \nabla_{\tilde{a}_{k}}+C_{k} \tilde{b}_{k} \cdot \nabla_{\tilde{b}_{k}}\right) \\
-\sum_{k, k^{\prime} \in \mathcal{K}} \operatorname{det}\left(k, k^{\prime}, \overrightarrow{b_{k}}\right)\left(\tilde{b}_{k} \cdot \nabla_{\tilde{a}_{k}}-\tilde{a}_{k} \cdot \nabla_{\tilde{b}_{k}}\right)
\end{gathered}
$$

Remark: As in [11] the explicit form of the dual of the generator implies that:

- $\nu$ is still the unique invariant measure of the diffusion $Y$ (the uniqueness is a consequence of the strong ellipticity of the generator).

- The symmetrization of $\tilde{\mathcal{L}}$ with respect to $\nu$ is exactly the generator of the OU process $X$.

The proof of the lemma is a straightforward application of the integration by parts formula. We skip the details. 
The symmetrized generator:

$$
\overline{\tilde{\mathcal{L}}}=\frac{1}{2} \sum_{k \in \mathcal{K}} \operatorname{tr}\left(\Sigma^{t} H_{\tilde{a}_{k}} \Sigma_{k}+\Sigma_{k}^{t} H_{\tilde{b}_{k}} \Sigma_{k}\right)-\sum_{k \in \mathcal{K}}\left(C_{k} \tilde{a}_{k} \cdot \nabla_{\tilde{a}_{k}}+C_{k} \tilde{b}_{k} \cdot \nabla_{\tilde{b}_{k}}\right)
$$

is a self adjoint nonpositive operator on $L_{2}\left(\mathbb{R}^{m}, d \nu\right)$ and it is know to have a spectral gap. In particular, there exists $\alpha>0$ such that:

$$
\inf _{\left\{f: \int f d \nu=0, \int f^{2} d \nu=1\right\}} \int f(-\overline{\tilde{\mathcal{L}}} f) d \nu=\alpha>0 .
$$

This property is crucial to our analysis. As in [11] we use it to derive the fact that, if $F \in$ $L_{2}\left(\mathbb{R}^{m}, d \nu\right)$ is such that $\int F d \nu=0$, then:

$$
\left\|e^{t \tilde{\mathcal{L}}} F\right\| \leq\|F\| e^{-\alpha t} .
$$

\section{Proof of the Main Result}

We first define a function $A$ on $\mathbb{R}^{m}$ with values in the space of $3 \times 3$ real matrices with trace 0 . To each element $y=\left(\tilde{a}_{k}, \tilde{b}_{k} ; k \in \mathcal{K}\right) \in \mathbb{R}^{m}$ we associate the matrice $A(y)$ defined by its entries:

$$
A(y)=\sum_{k \in \mathcal{K}}-\left(\tilde{a}_{k} \times k\right) \cdot k .
$$

Notice that, with this definition we have $A(Y(t))=\nabla \overrightarrow{\mathbf{v}}\left(t, x_{t}\right)$. This implies that, if we choose the initial condition $M_{0}=I$, then the solution of the linear system (8) is the Jacobian flow $\nabla \varphi_{0, t}(x)$. If the linear span of $\mathcal{K}$ is the whole $\mathbb{R}^{3}$, then an elementary (though tedious) computation shows that the hypoelliptic condition is satisfied and consequently, using the result recalled in Lemma 2.1 we see that there exist constants $\mu_{1} \geq \mu_{2} \geq \mu_{3}$ such that for any linearly independent fixed unit vectors $v_{1}, v_{2}, v_{3}$ in $\mathbb{R}^{3}$ we have almost surely

$$
\lim _{t \rightarrow \infty} \frac{1}{t} \ln \left|\nabla \varphi_{0, t}(x) w_{1} \wedge \cdots \wedge \nabla \varphi_{0, t}(x) w_{i}\right|=\mu_{1}+\cdots+\mu_{i}
$$

for any $1 \leq i \leq 3$. Notice that the Lyapunov exponents $\mu_{i}$ do not depend upon the starting point $x$. Indeed, they depend only upon the distribution of the stationary sequence $M_{t}$ and the latter does not depend upon the particular choice of $x$. Moreover since the velocity field is assumed to be incompressible, volume elements are preserved by the flow and in particular:

$$
\left|\nabla \varphi_{0, t}(x) e_{1} \wedge \nabla \varphi_{0, t}(x) e_{2} \wedge \nabla \varphi_{0, t}(x) e_{3}\right| \equiv 1,
$$

if we use the notation $e_{1}, e_{2}$ and $e_{3}$ for the vectors of the canonical basis of $\mathbb{R}^{3}$. In particular, this implies that:

$$
\sum_{i=1}^{3} \mu_{i}=0 .
$$

Given all that, the following corollary is an immediate consequence of Lemma 2.1.

Corollary 4.1 If the linear span of $\mathcal{K}$ is the whole $\mathbb{R}^{3}$ then the almost sure behavior of the surface area $\left|S_{t}\right|=\left|\varphi_{0, t}\left(S_{0}\right)\right|$ is given by:

$$
\liminf _{t \rightarrow \infty} \frac{1}{t} \ln \left|S_{t}\right| \geq \mu_{1}+\mu_{2}=-\mu_{3} .
$$


Proof:

Notice first that formulas (6) and (7) give:

$$
\left|\varphi_{0, t}\left(S_{0}\right)\right|=\int_{[0,1] \times[0,1]}\left|\nabla \varphi_{0, t}(S(\alpha, \beta)) v_{\alpha}(\alpha, \beta) \wedge \nabla \varphi_{0, t}(S(\alpha, \beta)) v_{\beta}(\alpha, \beta)\right| d \alpha d \beta,
$$

where:

$$
v_{\alpha}(\alpha, \beta)=\left[\frac{\partial S^{1}(\alpha, \beta)}{\partial \alpha}, \frac{\partial S^{2}(\alpha, \beta)}{\partial \alpha}, \frac{\partial S^{3}(\alpha, \beta)}{\partial \alpha}\right]^{t}
$$

and

$$
v_{\beta}(\alpha, \beta)=\left[\frac{\partial S^{1}(\alpha, \beta)}{\partial \beta}, \frac{\partial S^{2}(\alpha, \beta)}{\partial \beta}, \frac{\left.\partial S^{(} \alpha, \beta\right) 3}{\partial \beta}\right]^{t} .
$$

Using Jensen's inequality and Fatou's lemma we get:

$$
\begin{aligned}
\liminf _{t \rightarrow \infty} & \frac{1}{t} \ln \left|\varphi_{0, t}\left(S_{0}\right)\right| \geq \\
& \int_{[0,1] \times[0,1]} \liminf _{t \rightarrow \infty} \frac{1}{t} \ln \left|\nabla \varphi_{0, t}(S(\alpha, \beta)) v_{\alpha}(\alpha, \beta) \wedge \nabla \varphi_{0, t}(S(\alpha, \beta)) v_{\beta}(\alpha, \beta)\right| d \alpha d \beta .
\end{aligned}
$$

For each $(\alpha, \beta) \in[0,1] \times[0,1]$ we set $x=S_{0}(\alpha, \beta)$ and we can apply the result (25) above. This gives the existence of an event $\Omega_{(\alpha, \beta)}$ of full probability measure on which (25) holds with $i=2$. Consequently, the event:

$$
\begin{aligned}
& \tilde{\Omega}=\{(\omega, \alpha, \beta) \in \Omega \times[0,1] \times[0,1] \\
&\left.\lim _{t \rightarrow \infty} \frac{1}{t} \ln \left|\nabla \varphi_{0, t}(S(\alpha, \beta)) v_{\alpha}(\alpha, \beta) \wedge \nabla \varphi_{0, t}(S(\alpha, \beta)) v_{\beta}(\alpha, \beta)\right|=\mu_{1}+\mu_{2}\right\}
\end{aligned}
$$

is of full $\mathbb{P} \otimes d \alpha \otimes d \beta$ - measure. Using Fubini's theorem one sees that, $\mathbb{P}$-almost surely in $\omega \in \Omega$ the set of couples $(\alpha . \beta)$ for which (25) holds is of full $d \alpha d \beta$ measure. Together with $(26)$, this proves the desired result.

At this point, it is obvious that we only need to prove $\mu_{3}<0$. Given the facts that $\mu_{1}+\mu_{2}+\mu_{3}=$ 0 and $\mu_{1} \geq \mu_{2} \geq \mu_{3}$, this is equivalent to establishing that not all Lyapunov exponents are same. In order to do this, we use Lemma 2.2.

Lemma 4.1 Let $k \in \mathbb{R}^{3}$ be nonzero, let $n$ be a unit vector that is orthogonal to $k$ and let $M$ be the $3 \times 3$ matrix $M=(n \times k) \cdot k^{\prime} . A=\exp M$ induces a map $\tilde{A} z=\|A z\|^{-1} A z$ on $S^{2}$, the unit sphere in $\mathbb{R}^{3}$. If $m$ is any probability measure on $S^{2}$ invariant under the action of $\tilde{A}$, then $m$ must be supported in the plane perpendicular to $k$.

Proof:

$M$ maps the subspace orthogonal to $k$ onto zero and $k$ to the direction of $n \times k$. Applying $A$ repeatedly we see that $(\tilde{A})^{n}\left(S^{2}\right)$ ocnverges toward the equator circle orthogonal to $k$.

Lemma 4.2 If $\mathcal{K}$ spans the whole space $\mathbb{R}^{3}$ there is no probability measure on $S^{2}$ which is left invariant by $v \hookrightarrow \Theta_{t} v=\left\|\nabla \varphi_{0, t}(x) v\right\|^{-1} \nabla \varphi_{0, t}(x) v$ almost surely. 
Proof:

Let $m$ be a probability measure on $S^{2}$ which is left invariant by all the $\Theta_{t}$ For any $k_{0} \in \mathcal{K}$ we select a unit a vector $h$ which is orthogonal to $k_{0}$. Since $Y(t)=\left\{\tilde{\vec{a}}_{k}(t), \tilde{\vec{b}}_{k}(t), k \in \mathcal{K}\right\}$ is strongly elliptic, then $\vec{a}_{k_{0}}(t)=n, \vec{a}_{k *}(t)=0, \vec{b}_{k}(t)=0$ for $k * \neq k_{0}$ is in the support of the diffusion. Therefore $A=\exp (M)$, where $M=(n \times k) \cdot k^{\prime}$, is in the support of $\nabla \phi_{1}$. Consequently $m$ is left invariant by $\tilde{A}$ (definied in the same way as in the previous lemma). By the previous lemma, $m$ must be supported by the big circle in $S^{2}$ that is orthogonal to $k_{0}$. Therefore $m$ must suport on the intersection of the all big circle in $S^{2}$ that are orthogonal to $k$ for any $k \in \mathcal{K}$ respectively. But since $\mathcal{K}$ spans $\mathbb{R}^{3}$, this intersection must be empty. This completes the proof.

Proof of the main theorem: As pointed out after the proof of Corollary 4.1, we only need to prove that $\mu_{1}>\mu_{3}$ and in order to do so, we can once more assume that the starting point $x \in \mathbb{R}^{3}$ is fixed once for all. If $\mu_{1}=\mu_{3}$. then lemma 2.2 implies the existence of a positive quadratic form(positive symmetric matrix) on $\mathbb{R}^{3}$, say $Q$, such that:

$$
Q(u, u)=Q\left(\nabla \varphi_{0, t}(x) u, \nabla \varphi_{0, t}(x) u\right)
$$

Notice that we used the fact that, since $\varphi_{0, t}$ preserves volume elements, we have $\operatorname{det} \nabla \varphi_{0, t}(x)=$ 1. Consequently, the Guassian distribution:

$$
G(d u)=\frac{1}{(2 \pi)^{\frac{3}{2}} \operatorname{det}(Q)} \exp [-Q(u, u)] d u
$$

is left invariant by $\nabla \varphi_{0, t}(x)$. Consequcely if we denote by $m$ the measure induced by $G(d u)$ on $S^{2}$, then $m$ is invariant under the action of all the $\Theta_{t}$ defined in the previous lemma. This is a contradiction which completes our proof.

\section{References}

[1] A. Antoniadis and R. Carmona (1985): Infinite Dimensional Ornstein Ulhenbeck Processes Probab. Th. Rel. Fields 74, 31-54.

[2] M. Avellaneda and A. Majda (1990): Mathematical models with exact renormalization for turbulent transport. Commun. Math. Phys., 131, 381-429.

[3] M. Avellaneda and A. Majda (1991): An integral representation and bounds on the effective diffusivity in passive advection by laminar and turbulent flows. Commun. Math. Phys., 138, 339-391.

[4] P. Baxendale and B. Rozovskii (1988): Dynamo Effect for Random Magnetic Fields (preprint)

[5] P. Bougerol(1988): Theoremes limites pour les systemes lineaires a coefficients markoviens, Probab. Th. Rel. Fields, 78 , 193-221.

[6] P. Bougerol(1988): Comparaison des exposants de Lyapounov des processus markoviens multiplicatifs. Ann. Inst. Henri Poincare, 24, 439-489. 
[7] P.H. Baxendale (1986): Asymptotic Behavior of Stochastic Flows of Diffeomorphisms: Two Case Studies. Proba. Th. Rel. Fields 73, 51-85.

[8] R. Carmona (1997): Transport properties of Gaussian Velosity Fields. First S.M.F Winter School in Random Media. Rennes 1994 in Real and Stochastic Analysis: Recent Advances. ed. M.M. Rao, CRC Press.

[9] R. Carmona, S. Grishin and S. Molchanov (1994): Massively Parallel Simulations of the Transport Properties of Gaussian Velocity Fields. in Mathematical Models for Oceanography, eds R. Adler, P.Muller, B. Rosovskii. Birkhauser.

[10] R. Carmona and J. Lacroix (1990): Spectral Theory of Random Schrödinger Operators. Birkhaüser, Boston.

[11] R. Carmona and L. Xu (1996): Homogenization for Time Dependent 2-D Incompressible Gaussian Flows. Ann. of Applied Prob. 7, 265-279.

[12] A. J. Chorin (1994): Vorticity and Turbulence. Springer-Verlag

[13] H. Furstenberg and H. Kesten (1960): Products of Random Matrices. Ann. Math. Statist., 31, 457-469.

[14] K. Itô and H.P. McKean, Jr. (1974): Diffusion Processes and their Sample Paths. Springer Verlag, New York, N.Y.

[15] H. Kunita (1990): Stochastic Flows and Stochastic Differential Equations. Cambridge Univ. Press. Boston, MA.

[16] Y. Le Jan (1984): On isotropic Brownian motions. Z. Wahrscheinlichkeitstheorie verw. Geb. 70, 609-620.

[17] Y. Le Jan (1991): Asymptotic Properties of Isotropic Brownian Flows. in Spatial Stochastic Processes, eds K.S. Alexander, J.C. Watkins, pp 219-232, Birkhaüser, Boston.

[18] A.D. Virtser (1979): On products of random matrixes and operators. Theory of Probability and its Applications, 24, 367-377.

[19] C.L. Zirbel (1993): Stochastic Flows: Dispersion of a Mass Distribution and Lagrangian Observations of a Random Field. Ph. D. Princeton. 